\title{
PERFIL EPIDEMIOLÓGICO DA MORTALIDADE MASCULINA: CONTRIBUIÇÕES PARA ENFERMAGEM*
}

\author{
Jeane Cristina Anschau Xavier de Oliveira ${ }^{1}$, Áurea Christina de Paula Correa ${ }^{2}$, Luanna Arruda e Silva ${ }^{3}$, Isabele \\ Torquato Mozer ${ }^{4}$, Renata Marien Knupp Medeiros ${ }^{1}$
}

\begin{abstract}
RESUMO: Este estudo teve por objetivo descrever a mortalidade da população masculina na faixa etária de 20 a 59 anos em Cuiabá/Mato Grosso, no período de 2002 a 2012, segundo as condições sociodemográficas, grupo de causas, ocorrência e assistência ao óbito. Estudo ecológico, constituído por 6.050 registros de óbitos de homens com idade entre 20 a 59 anos, residentes em Cuiabá/Mato Grosso. Realizada análise descritiva com frequência absoluta e relativa e cálculo de coeficientes de mortalidade padronizados.Verificou-se que os homens solteiros, pardos, com idade entre 20 a 49 anos e com escolaridade inferior a 11 anos morrem devido a agravos relacionados a Causas Externas, Doenças do Aparelho Circulatório e Neoplasias Malignas. Considerando a maior frequência de mortes masculinas por causas evitáveis, destaca-se a importância de investimentos em promoção da saúde e prevenção de agravos na atenção primária. Evidenciou-se baixa qualidade nos registros por incompletude dos dados disponíveis.
\end{abstract}

DESCRITORES: Mortalidade; Saúde do homem; Enfermagem; Epidemiologia.

\section{EPIDEMIOLOGICAL PROFILE OF MALE MORTALITY: CONTRIBUTIONS TO NURSING}

ABSTRACT: The objective in this study was to describe the mortality of the male population in the age range from 20 to 59 years in Cuiabá/Mato Grosso between 2002 and 2012, according to the sociodemographic conditions, group of causes, occurrence and assistance to death. Ecological study, consisting of 6,050 death records of men between 20 and 59 years of age, living in Cuiabá/Mato Grosso. Descriptive analysis with absolute and relative frequencies was applied and the standardized mortality coefficients were calculated. It was verified that the single, mulatto men between 20 and 49 years of age with less than 11 years of education die due to problems related to External Causes, Diseases of the Circulatory Apparatus and Malign Neoplasms. In view of the higher frequency of male deaths due to avoidable causes, the importance of investing in health promotion and disease prevention in primary health care is highlighted. The low quality of the records was evidenced, due to incompleteness of the available data.

DESCRIPTORS: Mortality; Men's health; Nursing; Epidemiology.

\section{PERFIL EPIDEMIOLÓGICO DE LA MORTALIDAD MASCULINA: CONTRIBUCIONES PARA ENFERMERÍA}

RESUMEN: Estudio cuyo objetivo fue describir la mortalidad de la población masculina en la franja etaria de 20 a 59 años en Cuiabá/ Mato Grosso, en el periodo de 2002 a 2012, de acuerdo a las condiciones sociodemográficas, grupo de causas, ocurrencia y asistencia a óbito. Fue un estudio ecológico, compuesto por 6.050 registros de óbitos de hombres con edad entre 20 y 59 años que viven en Cuiabá/Mato Grosso. Se realizó el análisis descriptivo con frecuencia absoluta y relativa, además de cálculo de coeficientes de mortalidad estandarizados. Se ha verificado que los hombres solteros, pardos, con edad entre 20 y 49 años y escolaridad inferior a 11 años mueren en razón de agravios referentes a Causas Externas, Enfermedades del Aparato Circulatorio y Neoplasias Malignas. Considerándose la mayor frecuencia de muertes masculinas por motivos evitables, se destaca la importancia de inversiones en promoción de salud así como en prevención de agravios en la atención primaria. Se constató poca calidad en los registros por escasez de datos disponibles.

DESCRIPTORES: Mortalidad; Salud del hombre; Enfermería; Epidemiología.

*Artigo extraído da dissertação intitulada: "Perfil Epidemiológico da Mortalidade Masculina em Cuiabá-MT, 2002-2012". Universidade Federal de Mato Grosso, 2016.

${ }^{1}$ Enfermeira. Doutoranda em Enfermagem. Universidade Federal de Mato Grosso. Cuiabá, MT, Brasil.

${ }^{2}$ Enfermeira. Doutora em Enfermagem. Docente do Programa de Pós-graduação em Enfermagem da Universidade Federal de Mato Grosso. Cuiabá, MT, Brasil.

${ }^{3}$ Enfermeira. Mestranda em Enfermagem. Universidade Federal de Mato Grosso. Cuiabá, MT, Brasil.

${ }^{4}$ Enfermeira. Mestre em Enfermagem. Docente de Enfermagem da Universidade Federal de Mato Grosso. Cuiabá, MT, Brasil

Autor Correspondente:

Recebido: $15 / 12 / 2016$

Jeane Cristina Anschau Xavier de Oliveira Fraga

Finalizado: $26 / 04 / 2017$

Universidade Federal de Mato Grosso

Rua C nº 65 - 78.050322 - Cuiabá, MT, Brasil

E-mail: ane.anschau@gmail.com 


\section{- INTRODUÇÃO}

Entende-se que ser homem é a representação de um papel repleto de acepções e sentidos, e a expressão de sua masculinidade corresponde, via de regra, ao modelo de masculinidade hegemônica ${ }^{(1)}$, em que o homem deve ser forte e invulnerável. Tais características condicionam a maioria dos homens a assumirem uma postura de baixo autocuidado e afastamento dos serviços de saúde, especialmente os da atenção primária, contribuindo desfavoravelmente na saúde dessa população, verificada pelos altos índices de morbimortalidade.

Diante desse cenário, em 2009, o Ministério da Saúde instituiu a Política Nacional de Atenção Integral à Saúde do Homem (PNAISH), que objetivou qualificar a assistência à saúde masculina, norteada pelo princípio da atenção integral, com vistas à melhoria das condições de saúde ${ }^{(2)}$.

Ao longo do processo de implantação da política, foram selecionados 26 municípios que apresentaram os maiores índices de mortalidade masculina no Brasil. Dentre eles, Cuiabá/Mato Grosso foi uma das capitais selecionadas. Ainda que os níveis de mortalidade neste município fossem elevados, durante o processo de implantação da política, não houveram estudos epidemiológicos que averiguassem as causas de morbimortalidade masculina no local ${ }^{(3)}$.

Estudo que analisou o processo de implantação da PNAISH no município apontou que, diferente de outros programas e políticas governamentais, os profissionais atuantes na Atenção Primária não receberam capacitação técnica para o atendimento qualificado à saúde do homem. Ademais, apesar de a política reconhecer as questões de gênero como base para ações voltadas a esse público, as propostas foram pautadas no modelo biomédico, tendo como principal foco questões relativas à prevenção do câncer de próstata ${ }^{(3)}$.

Pesquisas demonstram que as taxas de mortalidade masculina continuaram crescentes mesmo após a implantação da PNAISH no território nacional, e as causas externas configuraram a principal causa de morte de homens na faixa etária de 20 a 59 anos, no país, no ano de $2010^{(3-4)}$. Tal dado demonstra a existência de contradição entre as ações ministeriais - voltadas à prevenção do câncer de próstata - e os dados epidemiológicos, demonstrando assim deficiência nas propostas apresentadas pela política.

Estudos têm apontado para a necessidade de propor ações que considerem as questões comportamentais e de gênero, a fim de atender as necessidades e especificidades da população masculina e diminuir seus índices de morbimortalidade, que atualmente são alarmantes quando comparado aos índices femininos, reflexo das desigualdades de gênero ${ }^{(5-6)}$.

Dessa maneira, ressalta-se a importância de conhecer o perfil de mortalidade masculina local e suas particularidades, com vistas a subsidiar a proposição de estratégias específicas para que a PNAISH, no nível da atenção primária à saúde, seja efetiva, ao implementar ações que permitam o alcance de suas metas.

Neste contexto, o enfermeiro é elemento essencial, uma vez que é um dos agentes promotores da saúde na atenção primária. Sendo assim, é importante que este profissional conheça as condições sociodemográficas e de saúde da população com vistas a promover ações de prevenção ligadas às principais causas de mortalidade masculina.

A partir dessa compreensão, o presente estudo teve como objetivo descrever a mortalidade da população masculina, na faixa-etária de 20 a 59 anos em Cuiabá, no período de 2002 a 2012, segundo as condições sociodemográficas, grupo de causas, ocorrência e assistência ao óbito.

\section{- MÉTODO}

Pesquisa do tipo ecológica, realizada a partir de dados de óbitos de homens de Cuiabá ocorridos entre 2002 a 2012, tendo como critério de inclusão: óbitos que apresentaram como local de residência e local de ocorrência do óbito o código do município de Cuiabá, na faixa etária de 20 a 59 anos. Os dados referentes aos óbitos foram coletados do Sistema de Informação sobre Mortalidade (SIM) do DATASUS, no primeiro semestre de 2015. Os dados populacionais foram obtidos pelo Instituto 
Brasileiro de Geografia e Estatística (IBGE), disponibilizados no site do DATASUS ${ }^{(7)}$.

As variáveis do estudo foram categorizadas em: Sociodemográficas (faixa etária, estado civil, raça/ cor e escolaridade); Grupos de causas: Doenças infecciosas e parasitárias - DIP (AOO - B99), Neoplasias Malignas - NM (C00-D48), Doenças do Aparelho Circulatório - DAC (100-199), Doenças do Aparelho Respiratório - DAR (J00-J99) e Causas Externas - CE (V01-Y98), classificados a partir das informações relativas à Causa básica do óbito de acordo com a Classificação Internacional de Doenças CID - $10^{a}$ revisão ${ }^{(8)}$ e categorizados de acordo com as fichas de qualificação dos Indicadores de Saúde organizadas pela Rede Intergerencial de Informações para Saúde - RIPSA ${ }^{(9)}$.

As variáveis relacionadas à Ocorrência do Óbito foram compostas pelos dados: Local de Ocorrência para todos os Grupos de causas, e especificamente para as Causas Externas foram obtidos os registros referente à Circunstância do Óbito e a Fonte de Informações. As variáveis relacionadas à Assistência ao Óbito foram compostas pelos dados: Assistência Médica; Realização de Exames; Realização de Cirurgia; Realização de Necropsia.

Para o processamento, gerenciamento e análise dos dados, foram utilizados os programas Microsoft Office Excel, Epilnfo 3.5.2 e Statistical Package for the Social Sciences-SPSS 20.

Realizou-se estatística descritiva com o cálculo da distribuição proporcional dos óbitos segundo as variáveis sociodemográficas e ocorrência do óbito. As variáveis relacionadas à assistência ao óbito foram distribuídas proporcionalmente em relação aos grupos de causas.

Os Coeficientes de Mortalidade Específico por Grupos de causas e Faixa etária foram obtidos por meio da fórmula: $\mathrm{CM}=\left[\left(\mathrm{N}^{\mathrm{o}}\right.\right.$ de óbitos pela causa $\mathrm{X}$, em determinado grupo etário, da área $\mathrm{A}$, período $\mathrm{t} \div$ População masculina do mesmo grupo etário, da área $\mathrm{A}$, no meio do período $\mathrm{t}) \times 100.000]^{(10)}$. Os Coeficientes de Mortalidade foram padronizados empregando-se o método direto ${ }^{(10)}$. Para analisar a variação percentual $(\Delta \%)$, foram utilizadas as proporções no início (2002) e no final (2012) do período, em que B é a proporção final e A é a proporção inicial, dada pela formula a seguir: $\Delta \%=(B-A) / A \times 100^{(11)}$.

Esta pesquisa foi aprovada pelo Comitê de Ética em Pesquisa do Hospital Universitário Júlio Muller sob o número CAAE: 41468814.7.0000.5541, parecer nº 953.428.

\section{- RESULTADOS}

A Tabela 1 apresenta os dados $(n=6050)$ relativos aos óbitos de homens segundo as variáveis sociodemográficas, ocorridos entre os anos de 2002 a 2012 em Cuiabá.

Tabela 1 - Distribuição da frequência $(n=6050)$, proporção (\%) e variação percentual $(\Delta \%)$ dos óbitos masculinos segundo as variáveis sociodemográficas. Cuiabá, MT, Brasil, 2002-2012 (continua)

\begin{tabular}{lccc} 
Variáveis & Frequência $(\mathbf{n})$ & Proporção $\mathbf{\%})$ & $\Delta^{\mathbf{1}}$ \\
\hline Faixa Etária & & & \\
\hline 20 a 29 anos & 1619 & 26,8 & $-10,3$ \\
\hline 30 a 39 anos & 1226 & 20,3 & -5 \\
\hline 40 a 49 anos & 1379 & 22,8 & $-15,8$ \\
\hline 50 a 59 anos & 1826 & 30,2 & $-34,4$ \\
\hline Raça/cor " & & & \\
\hline Branca & 1523 & 25,2 & $-30,2$ \\
\hline Preta & 555 & 9,2 & 39,4 \\
\hline Amarela & 11 & 0,2 & -50 \\
\hline Parda & 3855 & 63,7 & 21,2 \\
\hline Indígena & 12 & 0,2 & - \\
\hline Estado Civil ${ }^{\prime \prime \prime}$ & & & 11,9 \\
\hline Solteiro & 3441 & 56,9 & \\
\hline
\end{tabular}




\begin{tabular}{lccc}
\hline Casado & 1797 & 29,7 & $-31,5$ \\
\hline Viúvo & 85 & 1,4 & 111,1 \\
\hline Separado & 345 & 5,7 & 94,8 \\
\hline União Consensual & 137 & 2,3 & 123 \\
\hline Nenhum & 332 & 5,5 & -30 \\
\hline Escolaridade $^{\mathrm{IV}}$ & & & \\
\hline 1 a 3 anos & 1043 & 17,2 & $-25,9$ \\
\hline 4 a 7 anos & 2179 & 36 & 21,6 \\
\hline 8 a 11 anos & 1581 & 26,1 & 42,7 \\
\hline 12 e mais & 574 & 9,5 & 28,7
\end{tabular}

Nota: I -Variação percentual entre 2002 e 2012; II - Excluído 94 (1,6\%) registros referentes aos campos não preenchidos; III -Excluído 81 (1,3\%) registros referentes aos campos não preenchidos e 164 (2,7\%) registros informados como ignorado; IVExcluído 116 (1,9\%) registros referentes aos campos não preenchidos e 225 (3,7\%) registros informados como ignorado.

A faixa etária entre 50 a 59 anos apresentou maior proporção de óbitos 30,2\% ( $n=1826$ ). Quanto à distribuição dos óbitos segundo a raça/cor, houve maior proporção de óbitos entre os homens pardos $63,7 \%(n=3855)$. Os homens solteiros foram os mais vitimados $56,9 \%(n=3441)$, apresentando um incremento em seu percentual de 11,9\% passando de 49,5\% ( $n=266)$ em 2002 para 55,4\% $(n=314)$ em 2012.

Referente à escolaridade, os homens que possuíam entre quatro a sete anos de estudo foram os mais afetados $36 \%(n=2179)$ e apresentaram um percentual de aumento de $21,6 \%$ no período de 2002 a 2012.

Houve maior frequência dos agravos relacionados às CE 49,3\% ( $n=2982)$ e com uma variação percentual $(\Delta \%)$ de $-2,6 \%$ ao ano. As DAC figuraram com $22,1 \%(n=1339)$ e $\Delta \%(-11,2)$ ao ano. Em terceira posição emergiram com maior frequência os óbitos por afecções relacionadas às $N M 13,5 \%(n=814)$ e $\Delta \%(88,1)$ ao ano.

A Tabela 2 apresenta a distribuição dos Coeficientes de Mortalidade Padronizados segundo a faixa etária e os Grupos de causas. Observa-se, na Tabela 2, que a faixa etária 20 a 29 anos apresentou maior risco de mortalidade pelas CE. As DAC e as NM representaram maior risco de mortalidade para os homens na faixa etária de 50 a 59 anos.

Tabela 2 - Coeficiente de mortalidade padronizado (por cem mil homens) segundo faixa etária e grupo de causa. Cuiabá, MT, Brasil, 2002- 2012

\section{Coeficiente de Mortalidade}

Causas Externas

Doenças do Aparelho Circulatório

Neoplasias Malignas

Doenças Infecciosas e Parasitárias

Doenças Aparelho Respiratório

\begin{tabular}{ccccc}
\multicolumn{5}{c}{ Faixa-Etária } \\
\cline { 2 - 5 } & $\mathbf{2 0}$ a $\mathbf{2 9}$ & $\mathbf{3 0}$ a 39 & $\mathbf{4 0}$ a $\mathbf{4 9}$ & $\mathbf{5 0}$ a $\mathbf{5 9}$ \\
\hline & 2713,1 & 1823,2 & 1525,4 & 1556,9 \\
\hline & 101,4 & 374,6 & 1271,2 & 3578,6 \\
\hline & 82,3 & 193,3 & 674,8 & 2347,8 \\
\hline & 151,2 & 403,3 & 549,2 & 771 \\
\hline & 51,7 & 131,2 & 307,6 & 771
\end{tabular}

Na Tabela 3 estão discriminados os dados relacionados à ocorrência do óbito. Verifica-se que a variável Local de Ocorrência apresenta a distribuição dos óbitos de todos os Grupos de Causas, já as variável Circunstância do Óbito e Fonte de Informações descrevem a distribuição dos óbitos somente para as CE. Verifica-se que $61,5 \%(n=3721)$ dos óbitos ocorreram em hospitais. Referente aos óbitos por CE, em sua grande maioria a principal circunstância do óbito foi o homicídio $26,9 \%$ ( $n=1630$ ) e como principal fonte de informações destacaram-se os boletins de ocorrência 34,5\% ( $n=2087)$ que também apresentaram um aumento da variação percentual em 128\% entre os anos 2002 a 2012. 
Tabela 3 - Distribuição da frequência ( $n=6050)$, proporção (\%) e variação percentual ( $\Delta \%$ ) dos óbitos masculinos segundo sua ocorrência. Cuiabá, MT, Brasil, 2002-2012

\begin{tabular}{lccc}
\hline Ocorrência do óbito & Frequência (n) & Proporção (\%) & $\Delta \mathbf{\%}^{\mathbf{1}}$ \\
\hline Local de Ocorrência do óbito & & & \\
\hline Hospital & 3721 & 61,5 & $-11,3$ \\
\hline Via Pública & 987 & 16,3 & $-1,8$ \\
\hline Domicílio & 769 & 12,7 & 44,5 \\
\hline Outros & 478 & 7,9 & 32,6 \\
\hline Outro estabelecimento de saúde & 89 & 1,5 & 1,15 \\
\hline Ignorado & 6 & 0,1 & -100 \\
\hline Circunstancia do óbito & & & \\
\hline Homicídio & 1630 & 26,9 & $-3,9$ \\
\hline Acidente & 1024 & 16,9 & $-13,4$ \\
\hline Ignorado & 155 & 2,6 & 50 \\
\hline Suicídio & 146 & 2,4 & 31,5 \\
\hline Outros & 19 & 0,3 & - \\
\hline Fonte de Informações & & & \\
\hline Boletim de ocorrência & 2087 & 34,5 & 128 \\
\hline Hospital & 201 & 3,3 & $-13,5$ \\
\hline Outra & 131 & 2,2 & - \\
\hline Ignorado & 128 & 2,1 & - \\
\hline Família & 17 & 0,3 & -100 \\
\hline
\end{tabular}

Nota: I - Variação percentual entre 2002 e 2012

Os dados relativos à assistência prestada até o momento do óbito estão apresentados na Tabela 4. Verifica-se grande proporção de casos ignorados ou sem registros referentes à informação "recebeu ou não Assistência Médica durante a doença/agravo que ocasionou a morte". O mesmo se verifica nas variáveis Realização de Exames e Realização de Cirurgia. A respeito da realização ou não de necropsia, houve maior proporção da realização desse procedimento para as mortes por CE 87,9\% ( $n=2621)$.

Tabela 4 - Distribuição da frequência $(n=6050)$ e proporção $(\%)$ dos óbitos por Grupos de causas segundo as variáveis relacionadas a assistência ao óbito. Cuiabá, MT, Brasil, 2002-2012 (continua)

\begin{tabular}{lccccc} 
Assistência ao óbito & $\begin{array}{c}\text { DIP } \\
\mathbf{n}(\mathbf{\%})\end{array}$ & $\begin{array}{c}\mathbf{N M} \\
\mathbf{n}(\mathbf{\%})\end{array}$ & $\begin{array}{c}\text { DAC } \\
\mathbf{n}(\mathbf{\%})\end{array}$ & $\begin{array}{c}\text { DAR } \\
\mathbf{n}(\mathbf{\%})\end{array}$ & $\begin{array}{c}\mathbf{C E} \\
\mathbf{n}(\mathbf{\%})\end{array}$ \\
\hline Assistência Médica & & & & \\
\hline Com & $296(51,1)$ & $449(55,2)$ & $585(43,7)$ & $142(42,3)$ & $435(14,6)$ \\
\hline Sem & $7(1,2)$ & $4(0,5)$ & $79(5,9)$ & $6(1,8)$ & $557(18,7)$ \\
\hline Ignorado & $37(6,4)$ & $75(9,2)$ & $142(10,6)$ & $25(7,4)$ & $548(18,4)$ \\
\hline Sem Registro & $239(41,4)$ & $286(35,1)$ & $533(39,8)$ & $163(48,5)$ & $1442(48,4)$ \\
\hline Realização de Exames & & & & \\
\hline Com & $298(51,5)$ & $406(49,9)$ & $420(31,4$ & $126(37,5)$ & $148(5)$ \\
\hline Sem & $65(11,2)$ & $64(7,9)$ & $326(24,3)$ & $64(19)$ & $1192(40)$ \\
\hline gnnorado & $72(12,4)$ & $96(11,8)$ & $182(13,6)$ & $40(11,9)$ & $507(17)$ \\
\hline Sem Registro & $144(24,9)$ & $248(30,5)$ & $411(30,7)$ & $106(31,5)$ & $1135(38,1)$ \\
\hline Realização de Cirurgia & & & & \\
\hline Com & $21(3,6)$ & $153(18,8)$ & $78(5,8)$ & $4(1,2)$ & $149(5)$ \\
\hline Sem & $321(55,4)$ & $276(33,9)$ & $652(48,7)$ & $185(6,8)$ & $1291(43,3)$ \\
\hline
\end{tabular}


Cogitare Enferm. (22)2: e49724, 2017

\begin{tabular}{lccccc}
\hline Ignorado & $82(14,2)$ & $130(16)$ & $189(14,1)$ & $40(11,9)$ & $440(14,8)$ \\
\hline Sem Registro & $155(26,8)$ & $255(31,3)$ & $420(31,4)$ & $107(31,8)$ & $1102(37)$ \\
\hline Realização de Necropsia & & & & \\
\hline Com & $20(3,5)$ & $15(1,8)$ & $204(15,2)$ & $54(16,1)$ & $2621(87,9)$ \\
\hline Sem & $494(85,3)$ & $728(89,4)$ & $1002(39)$ & $242(72)$ & $101(3,4)$ \\
\hline Ignorado & $1(0,2)$ & $4(0,5)$ & $0(0)$ & $0(0)$ & $3(0,1)$ \\
\hline Sem Registro & $64(11,1)$ & $67(8,2)$ & $133(9,9)$ & $40(11,9)$ & $257(8,6)$
\end{tabular}

Legenda: DIP - Doenças infecciosas e parasitárias; NM - Neoplasias Malignas; DAC - Doenças do Aparelho Circulatório; DAR - Doenças do Aparelho Respiratório; CE - Causas Externas.

\section{- DISCUSSÃO}

No estado de São Paulo, entre o período de 1980 a 2009, observou-se a diminuição dos óbitos masculinos nos grupos etários mais jovens, com redução maior no grupo de 10 a 19 anos (32\%) e de 20 a 39 anos (10\%), enquanto na faixa etária de 40 a 59 anos houve aumento de $13 \%{ }^{(12)}$.

Ao contrário do que se observou em Cuiabá, a mortalidade masculina no Canadá apresenta comportamento distinto. Em 2011, a idade com maior registro de óbitos foi de 85 anos para homens e 89 anos para as mulheres. Foram poucas mortes observadas para ambos os sexos entre a população jovem $^{(13)}$, em contraponto com a realidade brasileira, em que morrem-se mais homens em idade jovem. Tais diferenças podem ser, em parte, explicadas pela concentração de ações canadenses na redução das mortes violentas entre adultos-jovens e pelo investimento no tratamento de doenças cardiovasculares. Destaca-se ainda questões culturais, como a similaridade de comportamento entre homens e mulheres, particularmente no que trata de hábitos de risco, como exposição ao tabagismo e estresse ocupacional ${ }^{(13)}$.

A raça/cor no Brasil é uma informação que passou a ser utilizada para medir a situação social dos indivíduos, bem como medir os riscos associados à sua saúde ${ }^{(14)}$, contudo, é importante ressaltar que inquéritos populacionais baseados no sistema classificatório do IBGE consideram a raça/cor autodeclarada, o que pode comprometer o estudo desse fenômeno.

Neste estudo, houve maior ocorrência de mortes para os homens da raça/cor parda, que pode estar relacionada às características da população mato-grossense, que é composta predominantemente pela população parda, como constatado pelos dados do IBGE no censo demográfico de 2010, quando $50,9 \%$ da população residente em Cuiabá pessoas que se autodeclaram da raça/cor parda ${ }^{(15)}$. Estudo realizado no Brasil no ano de 2012 sobre a mortalidade por homicídios, identificou que nos anos de 2002 a 2010 houve tendência crescente de mortalidade seletiva, com aumento das taxas de homicídios $(5,6 \%)$ para a população negra e forte queda $(24,8 \%)$ das taxas de homicídios na população branca ${ }^{(16)}, \mathrm{o}$ que sugere uma situação de vulnerabilidade da população negra no contexto brasileiro.

Em relação ao estado civil, em Cuiabá a maior proporção de óbitos ocorreu entre os homens solteiros. Na Dinamarca, um estudo realizado com 6,5 milhões de dinamarqueses, entre os anos de 1982 a 2011, com o objetivo de relacionar a mortalidade masculina e o estado marital, constatou que o risco de mortalidade entre homens solteiros foi maior em todas as faixas etárias analisadas, quando comparado com homens casados, divorciados e viúvos. Como possíveis justificativas para esses resultados, os autores do estudo apontaram: o benefício financeiro proporcionado pela união conjugal; o estilo de vida mais saudável entre os casados; bem como a maior inclusão social verificada entre esses indivíduos ${ }^{(17)}$.

Referente à escolaridade, este estudo apresentou maior proporção de mortes entre os homens com níveis de estudo inferiores a 11 anos. Pesquisa realizada no ano de 2010 com o objetivo de estimar os diferenciais de mortalidade por nível de escolaridade da população adulta brasileira, mostra que a probabilidade de morte de homens é menor entre os que possuem maiores níveis de escolaridade, em todas as regiões do país. Tais resultados podem ser explicados pelas desigualdades econômicas e sociais que resultam em diferenças no acesso à saúde, estilo de vida, hábitos alimentares e perfil 
epidemiológico em determinados grupos ${ }^{(4)}$.

A análise da mortalidade masculina, segundo os grupos de causas, revelou que em Cuiabá, entre as causas de mortes em homens, destacam-se em primeiro lugar as CE, seguido pelas DAC e em terceiro lugar as NM. Ao verificar os dados do perfil da situação da saúde do homem no Brasil, publicado pelo Ministério da Saúde, verifica-se que o panorama nacional se assemelha ao observado neste estudo: no Brasil, no ano de 2010, as CE representaram 35,2\% das mortes, as DAC 17,9\% e as NM 11,8\% na população masculina de 20 a 59 anos $^{(18)}$.

Panorama diferente foi apresentado pela capital do Estado de São Paulo em 2011, em que empatadas como primeira causa de mortalidade masculina ficaram as DAC $(25,52 \%)$ e CE (também com $25,52 \%)^{(17)}$.

Sobre os dados relativos ao Local de Ocorrência dos óbitos, em Cuiabá a maior parte deles ocorreram em hospitais. Foram poucos os registros com essa informação ignorada, o que possibilita a identificação de como foi prestada assistência ao indivíduo no momento do óbito ${ }^{(19)}$. Considerando que as principais causas de mortalidade masculina se referem a causas graves, que necessitam de internação hospitalar, é coerente que a maioria dos óbitos ocorra nesse local.

No que trata dos resultados sobre a Circunstância do Óbito, especificamente para as mortes por $\mathrm{CE}$, observou-se que a causas mais prevalentes foram os homicídios, seguidos dos acidentes, tendo como fonte de informação o boletim de ocorrência. Tais resultados corroboram com estudo realizado em Fortaleza-CE no ano de 2010, que também identificou como circunstância mais prevalente os homicídios 410 (19,4\%), seguidos pelos acidentes 181 (8,5\%) e tiveram o boletim de ocorrência como principal fonte de informação $575(27,2 \%)^{(20)}$.

Assim, é esperado que as circunstâncias mais observadas de mortes estejam relacionadas aos homicídios e acidentes de trânsito, visto que essas são as principais causas de mortalidade no Grupos de $\mathrm{CE}^{(21)}$. Ademais, é compreensível que a maioria dos registros tenham como fonte de informação os boletins de ocorrência. Com relação à variação percentual observada no variável boletim de ocorrência, era de se esperar um aumento considerável, visto que, em se tratando de óbitos por homicídios e acidentes de trânsito, tem-se a necessidade do registro da ocorrência e encaminhamento ao Instituto Médico Legal (IML).

Em Cuiabá no período estudado, houve a incidência de um percentual representativo de mortes sem assistência médica com destaque para as mortes por CE557 (18,7\%). Considerando os registros ignorados e os registros sem informação, o cenário se torna ainda mais preocupante, verificando-se um aumento significativo de óbitos sem atendimento médico ou sem essa informação, em comparação com os óbitos que possuem a declaração de recebimento de assistência.

Pesquisa que analisou a qualidade das informações de óbitos por CE em Fortaleza-CE apontou que em $64,96 \%$ dos registros de óbitos não houve preenchimento nos campos relativos à obtenção de assistência médica ${ }^{(22)}$, o que torna difícil distinguir se foram óbitos por mortes súbitas ou instantâneas, ocorridas sem tempo para atendimento médico, ou se foram outras as razões para a não assistência ${ }^{(21)}$.

A ocorrência de número relativamente alto de óbitos sem assistência impossibilita a identificação da causa básica de morte, o que pode ser considerado como fator dificultador da qualidade das estatísticas de mortalidade no país ${ }^{(23)}$.

Em relação à realização de necropsia, foi observado que em Cuiabá, a maioria dos casos em que foi realizada, decorreu dos óbitos por CE. Esse resultado pode ser esperado, uma vez que as mortes por causas violentas devem obrigatoriamente ser encaminhadas para o IML para realização desse procedimento $^{(24)}$.

Considerando que a realização da necropsia permite a obtenção de diagnósticos mais precisos e a avaliação da qualidade do atendimento prestado, a realização desse procedimento torna-se fundamental, não só para as mortes decorrentes de $\mathrm{CE}$, mas também para as mortes decorrentes de outras causas, uma vez que o acesso a dados confiáveis auxilia no planejamento de políticas de saúde e ações de vigilância epidemiológica. Contudo, é preciso ressaltar que os altos custos decorrentes da realização da necropsia configuram fator limitante para sua ampla efetivação.

Nesta pesquisa constatou-se grande proporção de dados não preenchidos e ignorados, 
principalmente os relativos à assistência prestada ao óbito. A subnotificação dos registros das Declarações de Óbito (DO) reforça a influência das desigualdades socioeconômicas e de acesso aos serviços de saúde, uma vez que o desconhecimento das características da pessoa que faleceu (sexo, idade, estado civil, e outras), da prestação de assistência médica adequada e da causa básica da morte dificultam a construção de indicadores que contribuam para o reconhecimento do perfil de mortalidade ${ }^{(25)}$. Tais informações permitem aos gestores e profissionais de saúde analisar a situação sanitária e propor ações de intervenção para a redução da mortalidade de populações específicas.

\section{CONSIDERAÇÕES FINAIS}

O perfil da mortalidade masculina em Cuiabá evidenciou que os homens solteiros, pardos, com idade entre 20 a 49 anos, com baixo nível escolar morreram devido aos agravos relacionados às $\mathrm{CE}$, DAC e NM.

Destaca-se que a incompletude no preenchimento dos dados referentes à assistência médica prestada no momento do óbito deixa dúvidas se o atendimento foi realizado ou se apenas não registrado. Nesse sentido, é recomendável que órgãos de vigilância em saúde do município de Cuiabá, os profissionais de saúde, entre estes médicos e enfermeiros, as instituições de ensino e os serviços de saúde, reconheçam a necessidade de melhoria da qualidade das informações dos registros dos óbitos, por meio da capacitação permanente de todos os envolvidos na geração dessas informações, na busca ativa das informações incompletas, assim como pelos óbitos não notificados.

Mediante a maior frequência de mortes masculinas por doenças crônicas, degenerativas e violentas, torna-se necessário recomendar que investimentos em promoção e prevenção da saúde; capacitação profissional; sensibilização da população masculina referente aos comportamentos de risco; educação em saúde nas escolas; entre outros, podem contribuir para mudança no panorama atual.

Nesse contexto, destaca-se a importância dos profissionais da saúde, em especial o enfermeiro, referência na atenção primária, que de posse dessas informações poderá agir com vistas a prevenir mortes e instituir ações de promoção e prevenção. Para além, destaca-se a sensibilização dos homens para acesso aos serviços de saúde, possibilitando diagnóstico precoce e contribuindo para a redução das mortes dessa população. Para tanto, é necessário investimento em recursos humanos e materiais, melhoria das condições de trabalho dos profissionais envolvidos com os cuidados dessa população, assim como a parceria com redes de apoio.

Entre as limitações da presente investigação, destaca-se a baixa qualidade dos registros, e a classificação dicotômica da DO e do SIM, pautadas no sexo biológico (feminino e masculino) não considerando as diversidades de gênero, o que gera subnotificação da informação dos índices de mortalidade nesses grupos. Nesse sentido, propõe-se discussões acerca da necessidade de inclusão dessas populações vulneráveis.

\section{REFERÊNCIAS}

1. Viana MER, Costa LMC, dos Santos RM, dos Anjos DS. O cuidado à saúde da população masculina em tempos de Política Nacional de Atenção Integral à Saúde do Homem: o que eles falam. Cultura de los Cuidados. [Internet] 2015;19(41) [acesso em 10 nov 2016]. Disponível: http://culturacuidados.ua.es/article/view/2015-n41-la-atencionde-la-salud-de-la-poblacion-masculina-en-los-tiempos-de-la-politica-nacional-de-atencion-integral-a-la-saludde-los-hombres-lo-que-ellos-hablan.

2. Ministério da Saúde (BR). Secretaria de Atenção à Saúde. Departamento de Ações Programáticas e Estratégicas. Política Nacional de Atenção Integral à Saúde do Homem: Princípios e Diretrizes. Brasília (DF): Ministério da Saúde; 2009.

3. Corrêa ACP, Mozer IT. Gestão do processo de implementação da política de saúde do homem. Revenferm UERJ. [Internet] 2016;24(1) [acesso em 10 nov 2016]. Disponível: http://dx.doi.org/10.12957/reuerj.2016.9483.

4. da Silva LE, Freire FHMA, Pereira RHM. Diferenciais de mortalidade por escolaridade da população adulta 
brasileira, em 2010. Cad. Saúde Pública. [Internet] 2016;32(4) [acesso em 10 nov 2016]. Disponível: http://dx.doi. org/10.1590/0102-311X00019815.

5. Vieira KLD, Gomes VLO, Borba MR, Costa CFS. Atendimento da população masculina em unidade básica saúde da família: motivos para a (não) procura. Esc. Anna Nery. [Internet] 2013;17(1) [acesso em 10 nov 2016]. Disponível: http://dx.doi.org/10.1590/S1414-81452013000100017.

6. Figueiredo WS, Schraiber LB. Concepções de gênero de homens usuários e profissionais de saúde de serviços de atenção primária e os possíveis impactos na saúde da população masculina. Ciênc. saúde coletiva. [Internet] 2011;16(Suppl1) [acesso em 23 out 2016]. Disponível: http://dx.doi.org/10.1590/S1413-81232011000700025.

7.Brasil. DATASUS. Arquivo base populacional para download. [Internet] 2016 [acesso em 13 dez 2016]. Disponível: http://www2.datasus.gov.br/DATASUS/index.php?area=0901\&item=1\&acao=35\&pad=31655.

8. Organização Mundial da Saúde.Classificação Estatística Internacional de Doenças e Problemas Relacionados à Saúde (CID-10). Centro Colaborador da OMS para a Classificação de Doenças em Português. $10^{a}$ revisão. $8^{a}$ ed. São Paulo: Editora da Universidade de São Paulo (EDUSP); 2000.

9. Ministério da Saúde (BR). Rede Intergerencial de Informações da Saúde. [Internet]Fichas de qualificação dos indicadores de saúde, 2012 [acesso em 17 de nov 2015]. Disponível: http://fichas.ripsa.org.br/2012/category/ mortalidade/?l=pt_B.

10. Laurenti R, Mello-Jorge MHP, Lebrão ML, Gotlieb SLD. Estatísticas de Saúde. $2^{a}$ ed. Revisada e atualizada. São Paulo: EPU; 2005.

11. de Souza TO, de Souza ER, PintoLW. Evolução da mortalidade por homicídio no Estado da Bahia, Brasil, no período de 1996 a 2010. Ciênc. saúde coletiva. [Internet] 2014;19(6) [acesso em 10 nov 2016]. Disponível: http:// dx.doi.org/10.1590/1413-81232014196.04772013.

12. Sala A, Mendes JDV. Perfil da mortalidade masculina no Estado de São Paulo. BEPA, Bol. epidemiol. paul. [Internet] 2010;7(82) [acessoem 10 nov 2016]. Disponível: http://periodicos.ses.sp.bvs.br/scielo.php?script=sci_ arttext\&pid=S1806-42722010001000003\&lng=pt.

13. Minister responsible for Statistics Canada. Mortality: Overview, 2010 and 2011. Report on the Demographic Situation in Canada.[Internet] 2013[acesso em 10 nov 2016]. Disponível: http://www.statcan.gc.ca/pub/91209-x/2013001/article/11867-eng.htm.

14. Rodrigues LC, Coelho TCB, dos Santos ABS, Peixoto MT, Góes SC. Desimportância da raça/cor e responsabilidade médico-legal no preenchimento da declaração de óbito. Rev. Baiana de Saúde Pública. [Internet] 2011;35(4) [acesso em 27 out 2016]. Disponível: http://inseer.ibict.br/rbsp/index.php/rbsp/article/view/261.

15. Instituto Brasileiro de Geografia e Estatística (IBGE). Ministério do Planejamento, Orçamento e Gestão. [Internet] Brasília (DF); 2014 [acesso em 10 nov 2016].Disponível: http://biblioteca.ibge.gov.br/index.php/ biblioteca-catalogo? view=detalhes\&id=264529.

16. Waiselfisz JJ. Mapa da Violência 2012: A Cor dos Homicídios no Brasil. [Internet] Rio de Janeiro: CEBELA, FLACSO; Brasília: SEPPIR/PR; 2012 [acesso em 10 nov 2016]. Disponível: http://www.mapadaviolencia.org.br/ mapa2012_cor.php.

17. Frisch M, Simonsen J. Marriage, cohabitation and mortality in Denmark: national cohort study of 6.5 million persons followed for up to three decades (1982-2011). IntJEpidemiol. [Internet] 2013;42(2) [acesso em 10 nov 2016]. Disponível: http://dx.doi.org/10.1093/ije/dyt024.

18. Ministério da Saúde (BR). Fundação Oswaldo Cruz, Instituto Fernandes Figueira. Perfil da situação de saúde do homem no Brasil. Rio de Janeiro (RJ): Ministério da Saúde; 2012.

19. da Cunha CC, Campos D, França EB. Uso da busca ativa de óbitos na avaliação do Sistema de Informações sobre Mortalidade em Minas Gerais, Brasil. Epidemiol.Serv. Saúde. [Internet] 2011;20(3) [acesso em 10 nov 2016]. Disponível: http://dx.doi.org/10.5123/S1679-49742011000300002.

20. de Andrade-Barbosa TL, Xavier-Gomes LM, Barbosa VA, Caldeira AP. Mortalidade masculina por causas externas em Minas Gerais, Brasil. Ciênc. saúde coletiva. [Internet] 2013;18(3) [acesso em 10 nov 2016]. Disponível: 
http://dx.doi.org/10.1590/S1413-81232013000300017.

21. Lessa I. Assistência Médica e Óbitos por Doença Arterial Coronariana no Brasil, 1980-1999. ArqBrasCardiol. [Internet] 2003;81(4) [acesso em 10 nov 2016]. Disponível: http://publicacoes.cardiol.br/abc/2003/8104/8104001. pdf.

22. Messias KLM, Bispo Junior JP, Pegado MFQ, Oliveira LC, Peixoto TG, Sales MAC, et al. Qualidade da informação dos óbitos por causas externas em Fortaleza, Ceará, Brasil. Ciêncsaúde coletiva [Internet] 2016;21(4) [acesso em 10 nov 2016]. Disponível: http://dx.doi.org/10.1590/1413-81232015214.07922015.

23. Santo AH. Causas mal definidas de mortes e óbitos sem assistência. Rev.Assoc.Med. Bras. [Internet] 2008;54(1) [acesso em 10 nov 2016]. Disponível: http://dx.doi.org/10.1590/S0104-42302008000100016.

24.Ministério da Saúde (BR). Secretaria de Vigilância em Saúde. Departamento de Análise de Situação de Saúde. Manual de preenchimento de declaração de óbito. Brasília: Ministério da Saúde; 2001.

25. Barbosa DA, Barbosa AMF. Avaliação da completude e consistência do banco de dados das hepatites virais no Estado de Pernambuco, Brasil, no período de 2007 a 2010. Epidemiol. Serv.Saúde. [Internet] 2013;22(1) [acesso em 10 nov 2016]. Disponível: http://dx.doi.org/10.5123/S1679-49742013000100005. 